\title{
Electrical Properties Of The OLED With Convergence Relation By Surface Treatment Methods
}

\section{Ho Shik Lee and Jae Hwan Kwon*}

Professor, Department of Health Administration, Dongshin University, Naju, 58245, Korea. *Professor, Graduate School of Education, Dongshin University, Naju, 58245, Korea.

*Corresponding author. Tel.: +82-010-3606-8686; Email address: jhkwon@ dsu.ac.kr

Article History:Received:11 november 2020; Accepted: 27 December 2020; Published online: 05 April 2021

Abstract: ITO (indium-tin-oxide) is used widely as the anode electrode in organic electroluminescence devices (OLEDs) Several studies have dealt with the effect of ITO chemical treatment but to the OLEDs performance have not been considered the effect of this treatment. We were present here results regarding these effect. We studies of the effect of surface treatments on the properties of ITO substrate. We were performed chemical treatment with RCA and Aqua regiaof the OELDs. Since we will understand the properties of OELDs, we were performed Fowler-Nordheim tunneling. The buffer layer and ITO surface treatment were performed to increase the efficiency of the OLEDs. As a result, we were obtained conform the surface properties of ITO can be significantly changed by chemical treatment. The oxygen decrease produced by chemical treatment lead to increase of oxygen vacancies. We conformed that the oxygen vacancies role on increasing carrier injection. A study on the cause of improving the performance of the device using the surface treatment and the usingthe buffer layer will be of great help in understanding the relationship between the properties of the ITO substrate and the organic electroluminescent device.

Keywords: Indium-tin-oxide (ITO), OLED, Fowler-Nordheim tunneling, RCA treatment, Aqua regia treatment.

\section{Introduction}

The improvement in luminous efficiency can be due to the possibility of manufacturing an amorphous organic thin film having good characteristics and thus improving the injection of electrons or holes. In the early development stage, the development of various luminous colors was the main focus, and studies on white luminescence [1-4] were actively conducted as an extension of these. Efforts to improve luminous efficiency were attempted at the early stage of the doping method, and then efforts were made through the development of high-luminance materials. As an understanding of the operatingprinciple of an organic electroluminescent device (OLED), a high-luminance light-emitting material using the same has been developed. However, since the development of light-emitting materials is slow and requires a lot of initial investment, researchers first understand the driving principle of the device, and from this, focus on finding necessary elements for improving device efficiency, and these efforts are classified as follows [5-8].

In order to improve the efficiency of an OLED, 1) improvement of transporter injection, 2) a structure of a device that can efficiently recombine with a numerical balance of electrons and holes, and 3) development of materials with high luminous efficiency are required $[9,10]$.

In this paper, among the various surface treatment methods of ITO substrates, the surface of the ITO substrate is stabilized through a chemical treatment and the efficiency of the OLED is improved by adjusting the surface characteristics suitable for organic electroluminescent devices. It was intended to investigate the phenomenon at the interface between the substrate and the organic material. RCA and Aqua regia solutions were used as a chemical treatment method to examine the changes in the characteristics of the device when the surface and composition of the ITO substrate was changed, and investigate how the surface changes affect the characteristics of the device.

\section{Materials}

As the material used in this experiment, $\mathrm{Alq}_{3}$, a metal complex compound, was used as a light emitting layer of an organic electroluminescent device. And diamine-based TPD was used as the hole transport layer. The molecular structure of TPD and $\mathrm{Alq}_{3}$ are shown in Figure 1. $\mathrm{Alq}_{3}$ is a chelate compound surrounded by an 8hydroxyquinoline ligand with a metal ion, $\mathrm{Al}$ as the center, and is a metal complex that has been studied since it was first known by Kodak. $\mathrm{Alq}_{3}$ is a green fluorescent material, has very high quantum efficiency, and has good luminescence properties as a light-emitting material because it is a very stable film formation, good transporter movement, and a good thermal resistor. TPD is a material having the most excellent properties among materials used as a hole transport layer, and the reason for using TPD and $\mathrm{Alq}_{3}$ is that these two materials are the most basic materials used in organic electroluminescent devices and exhibit good properties.

*Corresponding author: Jae Hwan Kwon

Professor, Graduate School of Education, Dongshin University, Naju, 58245, Korea.

Email address: jhkwon@dsu.ac.kr 


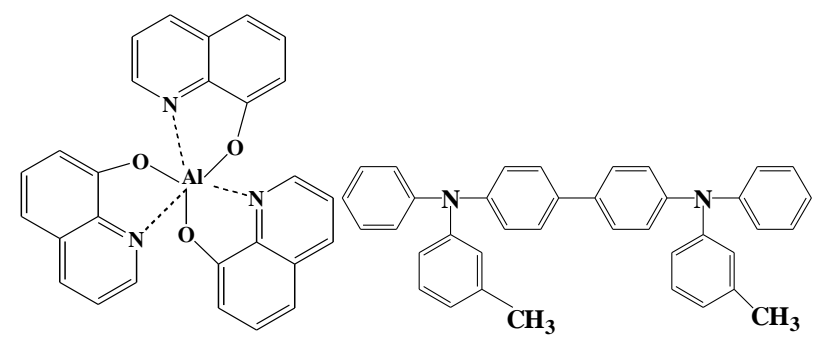

(a) $\mathrm{Alq}_{3}$

(b) TPD

Figure 1Molecular structure

\section{Results}

\subsection{C-V (Cyclic-Voltammetry) Characteristics}

Cyclic Voltammetry was used to measure the energy gap of the organic material in this experiment. Figures 2 show the results of measuring the cyclic voltammetry of the $\mathrm{Alq}_{3}$ and TPD used in the experiment. By knowing the energy gap of a material by cyclic voltammetry, it is possible to confirm the process by which the carrier can inject more efficiently and increase the luminous efficiency. By measuring the voltage at which oxidation and reduction of a material begins by circulating voltage current and adding $4.87 \mathrm{eV}$ which is the correction value of the cyclic voltammetry for the UPS, we can found the ionization energy and electron affinity of the material.From the result of measuring the cyclic voltammetry, it can be seen that in the case of TPD, the oxidation reaction is $+0.67 \mathrm{eV}$ and the reduction reaction is $-2.30 \mathrm{eV}$. Therefore, adding the correction value of $4.8 \mathrm{eV}$ results in ionization energy of $5.47 \mathrm{eV}$ and an electron affinity of $2.50 \mathrm{eV}$. As a result, it can be confirmed that the energy gap of TPD is $2.97 \mathrm{eV}$, which is the difference between ionization energy and electron affinity. In the same way, in the case of $\mathrm{Alq}_{3}$, the oxidation reaction is $+0.98 \mathrm{eV}$, the reduction reaction is $-1.66 \mathrm{eV}$, the ionization energy is $5.78 \mathrm{eV}$, the electron affinity is $3.14 \mathrm{eV}$, and the energy gap is $2.64 \mathrm{eV}$.
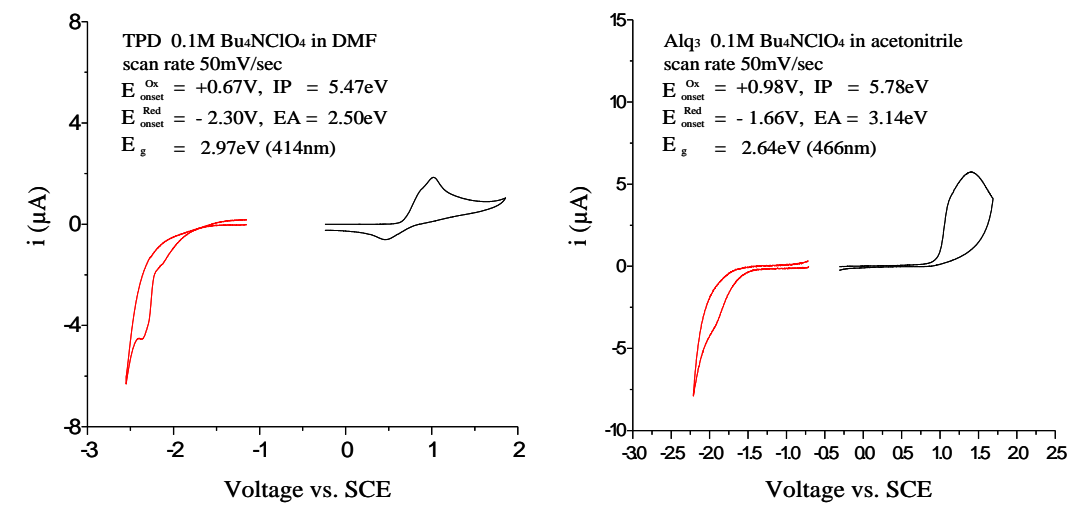

(a)TPD (b) $\mathrm{Alq}_{3}$

Fiurure 2Cyclic Voltammogram of the (a) TPD and (b) $\mathrm{Alq}_{3}$.

\subsection{XPS and Binding Energy spectra of the ITO}

Figure 3 shows the results of XPS (X-ray photoelectron spectroscopy) analysis of the ITO. In Figure 3, the constituents of ITO without surface treatment and the mostly indium (In), tin (Sn), and oxygen (O), and the surface contains carbon (C) due to residual organic matter. In the case of indium, the spectrum appears at 444.8 $\mathrm{eV}$ and the spectrum appears at $452.4 \mathrm{eV}$. Each spectrum represents a spectrum of photoelectrons from In $3 \mathrm{~d}_{5 / 2}$ and In $3 \mathrm{~d}_{3 / 2}$. Looking at the spectrum of the In $3 \mathrm{~d}$ at the surface and bulk of ITO, respectively, there is no change in the binding energy between the surface and the bulk. The spectrum due to the photoelectrons of $\mathrm{Sn} 3 \mathrm{~d}$ appears from the tin. At $486 \mathrm{eV}$, the spectrum due to $\mathrm{Sn} 3 \mathrm{~d}_{5 / 2}$ and the spectrum in the binding energy region due to electrons of $\mathrm{Sn} 3 \mathrm{~d}_{3 / 2}$ are observed. Like indium, tin does not show any change in the spectrum of the surface and bulk. The absence of these spectral changes indicates that no structural defects or new bonds are created. The component that shows the greatest change in binding energy in ITO is oxygen. In the case of oxygen, a change in the spectrum between the bulk and the surface appears due to a new bond with external hydrogen. The oxygen spectrum appears in the binding energy region corresponding to the photoelectron of $\mathrm{O} 1 \mathrm{~s}$. As shown in Figure 4, the surface shows a change in spectrum due to the influence of the new bond.As shown in Figure 4, there are three types of oxygen present on the surface. The first $\left(\mathrm{O}_{\mathrm{I}}\right)$ is the binding energy by $\mathrm{O}_{2-}(530.2 \mathrm{eV})$, which means the binding energy by oxygen that is perfectly bonded. The second $\left(\mathrm{O}_{11}\right)$ is the binding energy by 
$\mathrm{O}_{2^{-}}(531.7 \mathrm{eV})$, which means the binding energy by photoelectrons of oxygen adjacent to the oxygen vacancy. Finally, the third $\left(\mathrm{O}_{\mathrm{III}}\right)$ means the binding energy $(533.4 \mathrm{eV})$ by oxygen $(-\mathrm{OH})$ bound to the surface hydrogen.

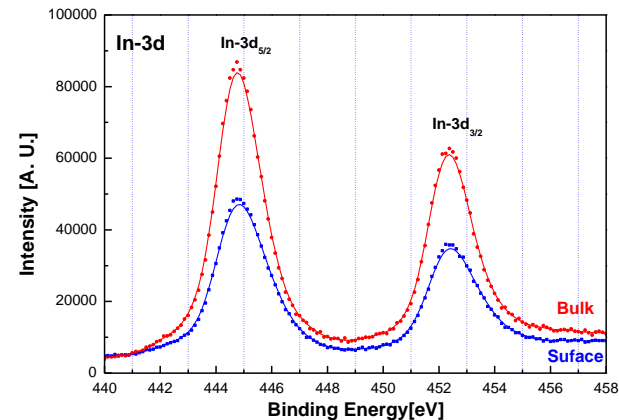

(a) In $3 d$ core level spectra

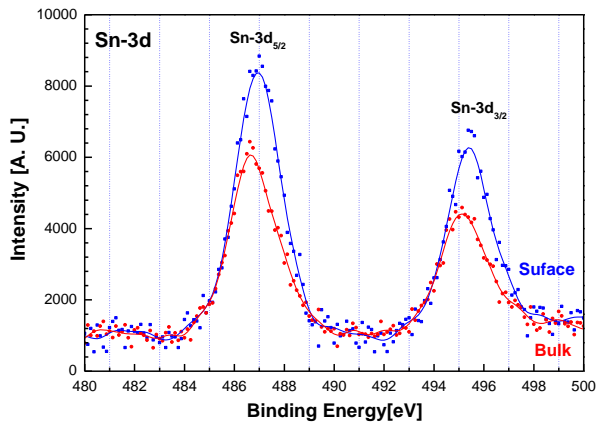

(b) Sn 3d core level spectra

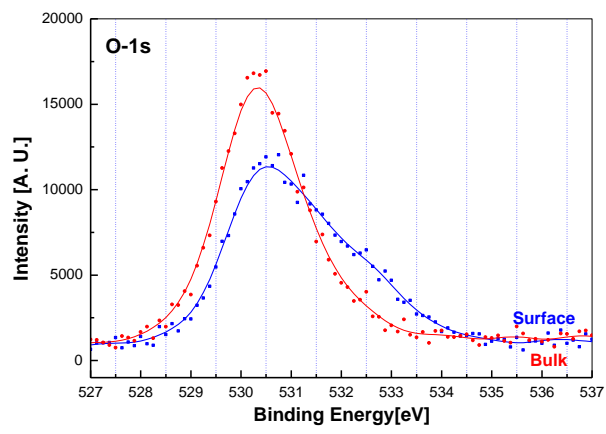

(c) $\mathrm{O} 1 \mathrm{~s}$ core level spectra

Fiurure 3XPS spectra of the ITO substrate.

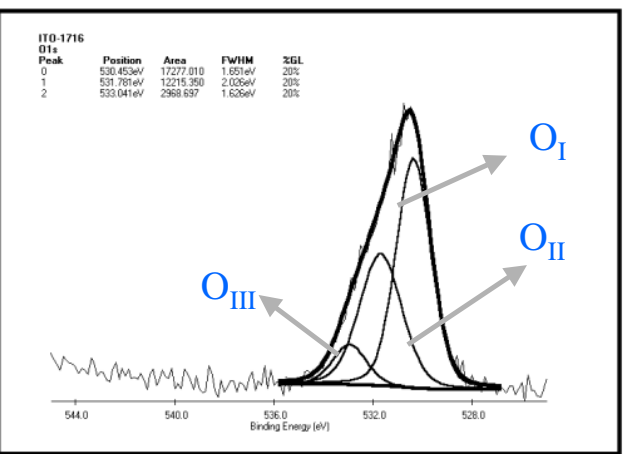

Fiurure 4Binding energy of oxygen species of the ITO suface.

\subsection{Chemical treatment( $R C A$, Aqua regia)}

There are two main types of chemical treatment methods. The first is a method of treating the ITO substrate by mixing aqueous ammonia, hydrogen peroxide, and secondary distilled water, and this method is called the RCA treatment method. The second method is to mix nitric acid, hydrochloric acid and secondary distilled water for treatment. The method of treating the surface with an acid is called Aqua regia treatment.

In the RCA solution, ammonia water $\left(\mathrm{NH}_{4} \mathrm{OH}\right)$, hydrogen peroxide $\left(\mathrm{H}_{2} \mathrm{O}_{2}\right)$, and secondary distilled water were mixed in a ratio of 1:4:25, and the ITO substrate was put in a state where the temperature was heated to $60^{\circ} \mathrm{C}$. The Aqua regia solution was prepared by mixing nitric acid $\left(\mathrm{HNO}_{3}\right)$, hydrochloric acid $(\mathrm{HCl})$, and secondary distilled water at a mixing ratio of 1:3:25, put an ITO substrate, treated the surface, and cleaned the device. In addition, the ITO substrate surface was treated alternately between the RCA treatment with the best device performance and the Aqua regia treatment with the best device performance. For the improvement of device performance by chemical treatment, the electrical characteristics are investigated through current densityvoltage, luminance-voltage, and Fowler-Nordheim tunneling, and surface characteristics are analyzed for the main cause that affects the actual device performance through the XPS.

\subsubsection{RCA treatment}


Figure 5 shows the voltage-current density curve of the device when the surface is not treated with RCA and when the surface is treated. It can be seen that when the RCA treatment was treated for 5 min and 10 min, the carrier injection characteristics were improved by 10 times compared to the case without treatment. It can be seen that the emission intensity of the device is further improved when the processing time is 5 and 10 min, compared to the case of not processing.

In the case of $5 \mathrm{~min}$ of RCA treatment at $10 \mathrm{~V}$, the current density is shown as a $20 \mathrm{~mA} / \mathrm{cm}^{2}$. Compared with the case of 10 min treatment, it could not be seen a little difference in the current density. However, it can be seen that there is a difference in luminance between the 5 min treatment and the $10 \mathrm{~min}$ treatment in the luminance characteristics. At $10 \mathrm{~V}$, the $5 \mathrm{~min}$ treatment is about $200 \mathrm{~cd} / \mathrm{m}^{2}$ and the $10 \mathrm{~min}$ treatment is $100 \mathrm{~cd} / \mathrm{m}^{2}$. The difference in luminance occurs at almost the same current density, indicating that the RCA 5 min treatment improved the efficiency. As a result, it was confirmed that the most efficient condition in the RCA treatment was 5 min treatment.

It can be seen that the cause of the device performance improvement according to the RCA process is due to the increase of the injected carriers as confirmed through the current density-voltage and luminance-voltage characteristics. To confirm this increase in transporter injection, it can be analyzed by applying the FowlerNordheim tunneling theory. Figure 6 shows a Fowler-Nordheim plot was plotted using the current densityvoltage curve obtained from the actual device.
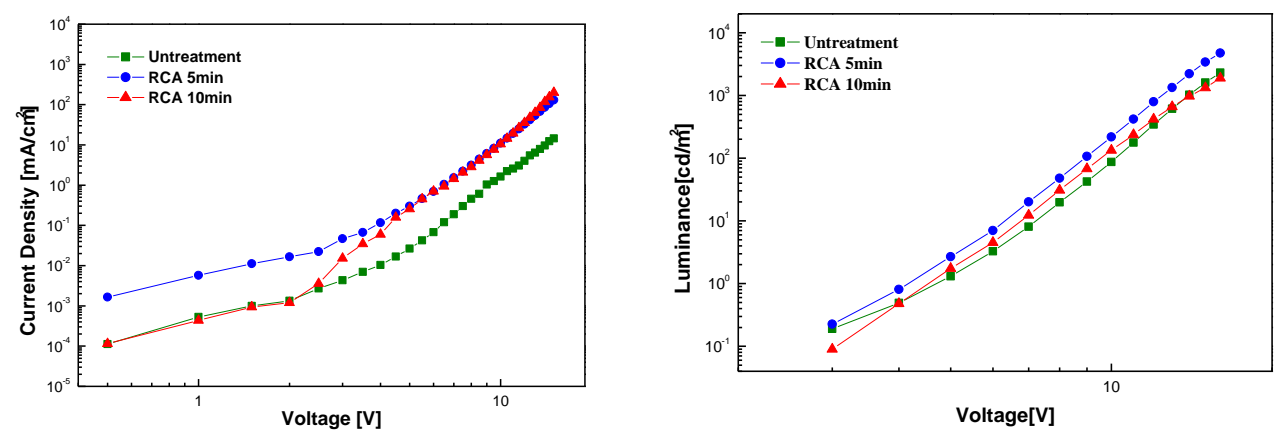

(a) Current density-voltage (b) luminance-voltage

Figure5Electrical Properties of the ITO substrates with RCA treatment.
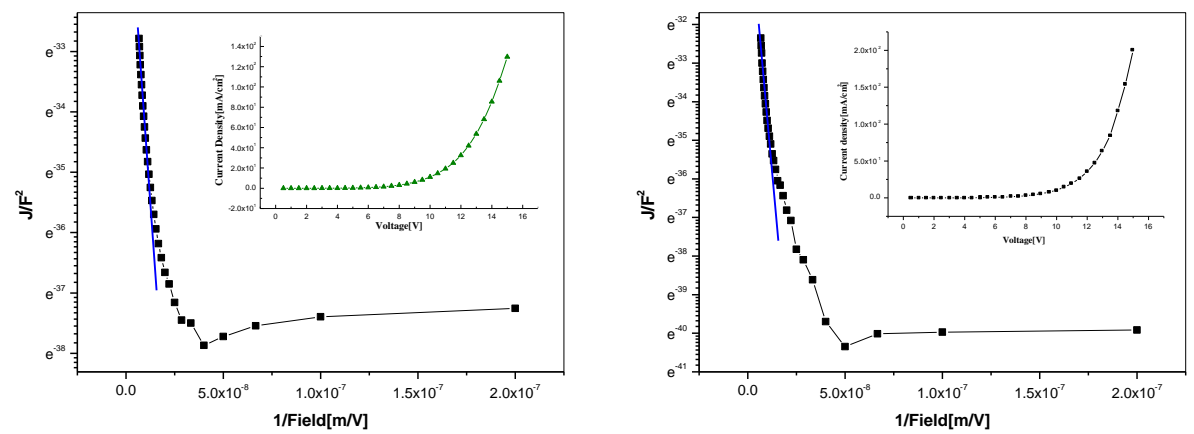

(a) RCA $5 \min ($ b) RCA 10 min

Figure6Fowler-Nordheim plot of the OLED with RCA treatment..

When the ITO surface was chemically treated, the spectrum of the surface and bulk of indium, tin, and oxygen is shown in Figure 7, 8, 8. It was confirmed that the surface and bulk of indium and tin did not change as shown in the spectrum. The biggest change that is consistent is the change in oxygen on the surface. In the case of chemical treatment as oxygen, the element that affects the electrical conductivity in the ITO substrate, it can be seen that the change in other composition is inconsistent. 

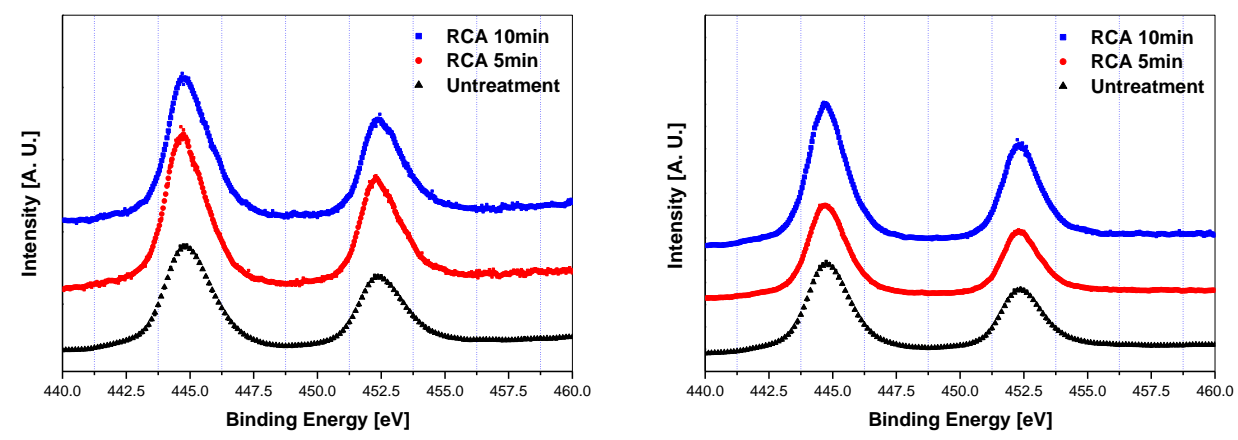

(a) On the surfac (b) In the bulk

Figure 7In 3d core-level XPS of the ITO with RCA treatment.

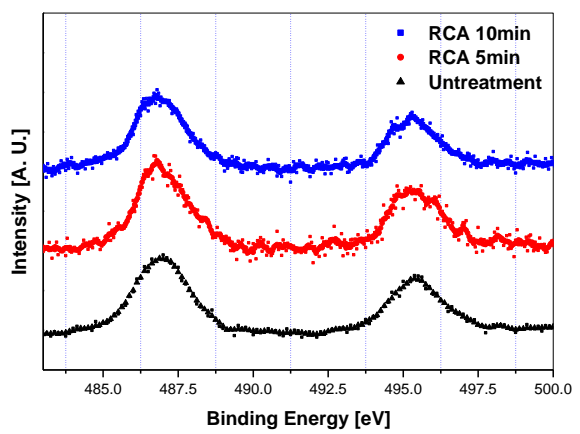

(a) On the surface

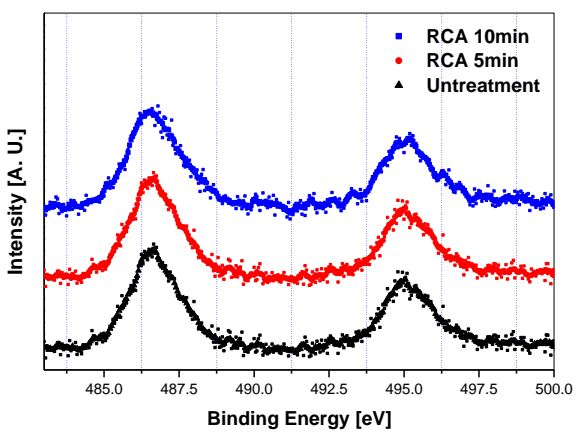

(b) In the bulk

Figure 8Sn 3d core-level XPS of the ITO with RCA treatment.

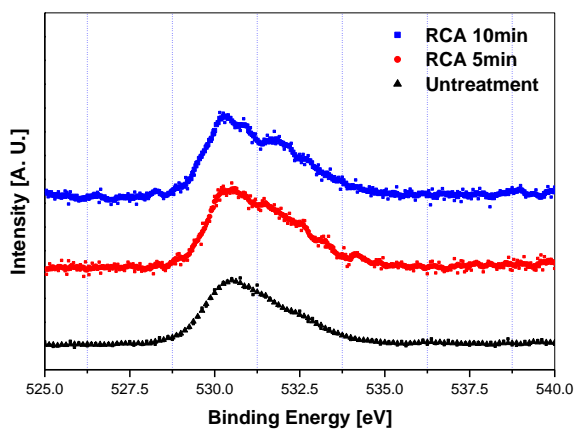

(a) On the surface

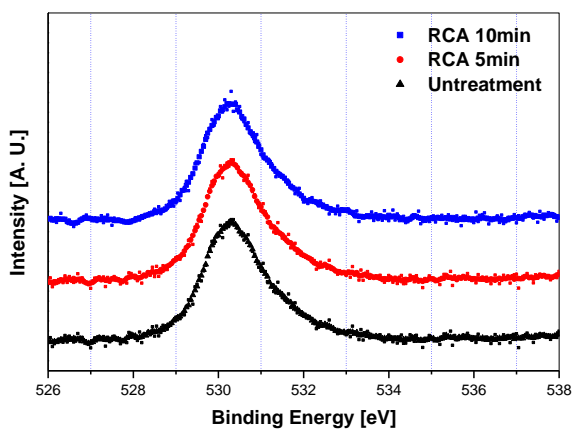

(b) In the bulk

Figure 901 s core-level XPS of the ITO with RCA treatment.

However, it can be seen that the change in the composition of oxygen is consistently changed depending on the treatment conditions. When comparing the performance of the device according to the change in the oxygen composition on the surface, it can be seen that the performance of the device is improved as the composition of oxygen decreases.Also, it was confirmed through device characteristics that the amount of holes injected increases according to the composition ratio of oxygen, and that the increased amount of holes contributes to light emission of the device.

\subsubsection{Aqua regia treatment}

Figure 10 shows the current density-voltage characteristics of the device, which appears when the device is directly manufactured by treating the surface of the ITO substrate. In the case of Aqua regia treatment, it can be confirmed through the current density-voltage characteristics that the injection of the carrier is very higher than that of the untreated device. Depending on the treatment time, in the case of $5 \mathrm{~min}$ and the injection of carriers into the device increased the most, followed by $10 \mathrm{~min}$.In the current density-voltage characteristics, the luminance-voltage characteristics also show that the amount of transporters contributing to direct light emission increases in proportion to the amount of carrier injected in the order of $5 \mathrm{~min}$ and $10 \mathrm{~min}$ depending on the processing time. We were obtained the current density value when treated for $5 \mathrm{~min}$ of Aqua regia at $10 \mathrm{~V}$ is 60 
$\mathrm{mA} / \mathrm{cm}^{2}$, and $30 \mathrm{~mA} / \mathrm{cm}^{2}$ when treated for $10 \mathrm{~min}$. When the treatment time was $5 \mathrm{~min}$, it was found that the current density increased by about 2 times. The luminance characteristic is $1000 \mathrm{~cd} / \mathrm{m}^{2}$ when 5 min treatment is performed at $10 \mathrm{~V}$, and $300 \mathrm{~cd} / \mathrm{m}^{2}$ when $10 \mathrm{~min}$ treatment is performed. It was confirmed that the luminance characteristics also increased with the increase of the injected transporter.

This is due to the variation of the effective potential barrier height obtained from the Fowler-Nordheim plot of the current density-voltage characteristic curve.Like the RCA treatment, the current density-voltage characteristics of the device treated with Aqua regia are also shown in Figure 11 and we were observed that it is well applied to the Fowler-Nordheim tunneling theory. When the Aqua regia treatment was performed rather than the RCA treatment, the operating voltage was slightly decreased. However, it was found that the operating voltage did not change according to the conditions of Aqua regia treatment.
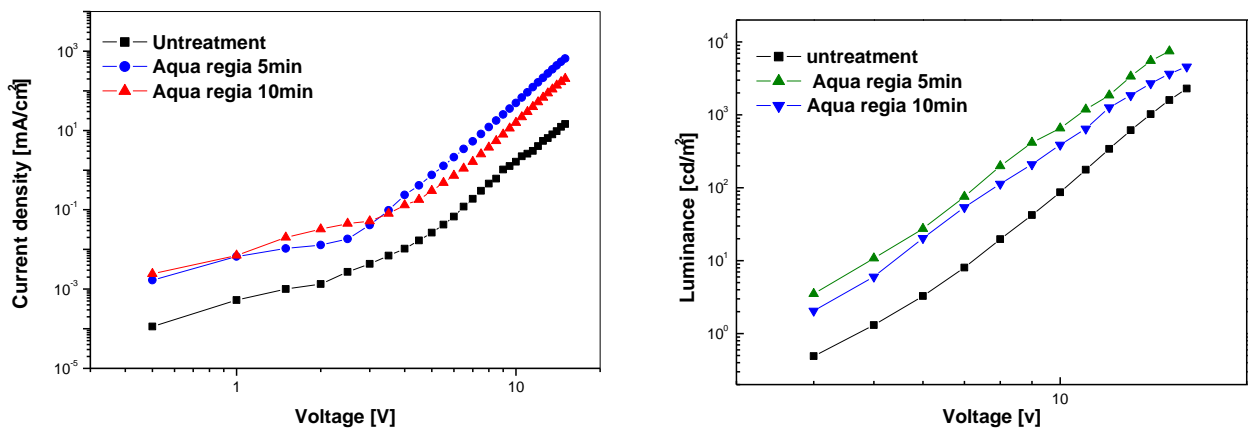

(a) Current density-voltage

(b) luminance-voltage

Figure 10Electrical Properties with ITO substrates of Aqua regia treatment.

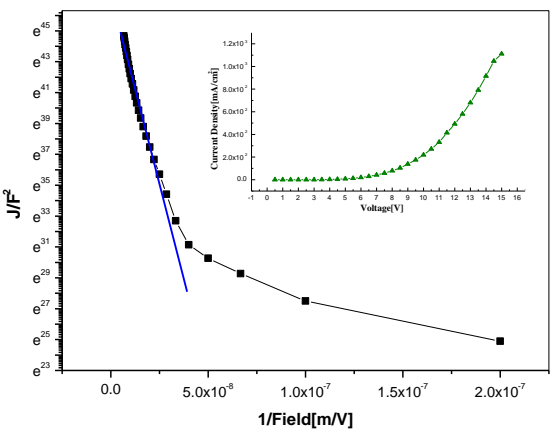

(a) Aqua regia $5 \mathrm{~min}$

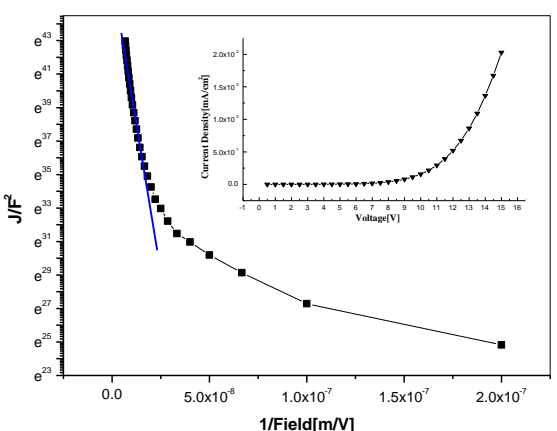

(b) Aqua regia 10min

Figure 11Fowler-Nordheim plot of the OLED with Aqua regia treatment.

\section{Conclusions}

Since the surface treatment of the ITO substrate is essential to improve the performance of the organic electroluminescent device, we tried to investigate the effect and cause of the surface characteristics of the ITO substrate on the performance of the device. The surface treatment of the ITO substrate was chemically treated (RCA, Aqua regia) to analyze the characteristics of the device.

The improvement of device performance by chemical treatment could result in an increase in transporter injection due to a decrease in the effective potential barrier. This phenomena was occurs when carrier flows at the interface between the ITO substrate and the organic material.In the case of RCA treatment, the height of the effective potential barrier decreased by $0.08 \mathrm{eV}$, and even in the case of Aqua regia treatment, $0.08 \mathrm{eV}$ was decreased.Also, in the case of mixing treatment, it was found that the most decrease was $0.14 \mathrm{eV}$.The carrier injection increases as the height of the effective potential barrier decreases, and the increase of the injected carrier improve the device performance.As for the composition change of the surface of the ITO substrate by chemical treatment, oxygen changed the most.It can be seen that the RCA treatment decreased by $5 \%$, the Aqua regia treatment decreased by $6 \%$, and the mixed treatment decreased by $11 \%$.

Also, it was confirmed that the $\mathrm{O}_{\mathrm{I}}$ component, which is the factor that most affects the device performance, decreased to $7 \%$ in the case of RCA treatment, $12 \%$ in the case of Aqua regia treatment, and $20 \%$ in the case of mixed treatment. The decrease in the $\mathrm{O}_{\mathrm{I}}$ component is the main reason for increasing the oxygen vacancy, and the increase in the oxygen vacancy increases the number of carriers.It can be concluded that an increase of carriers injected into the organic material consequently improves the device performance. 


\section{References}

1. Kallmann. H,Pope. M. Preparation of Thin Anthracene Single Crystals. Review of Scientific Instruments.1958;29:11993-994.

2. M. G. Mason, C. W. Tang. \&L. S. Hung, et al. Interfacial chemistry of $\mathrm{Alq}_{3}$ and $\mathrm{LiF}$ with reactive metals. Journal of Applied Physics.2001;89:52756-2765.

3. Sun Zhengyi, Ding Xumm in, Ding Baofu, et. al. Buffer enhanced electron injection in organic lightemitting devices with copper cathode. Organic Electrons.2013;14:2 511-515.

4. Seong Shan Yap, Ren Bin Yang, ThianKhok Yong, et al,.Effect of diamond-like carbon in TPS-Alq doped PVK organic light-emitting devices. Diamond and Related Materials. 2008;18:2380-383.

5. Kannan Seshadri, C. Daniel Frisbie. Potentiometry of an operating organic semiconductor field-effect transistor.Applied Physics Letter.2001;78:7993-995.

6. Hongmei Zhang, Yanfeng Dai, Dongge Ma, et. al.High Efficiency tandem organic light-emitting devices with $\mathrm{Al} / \mathrm{WO}_{3} / \mathrm{Au}$ interconnecting layer, Applied Physics Letter.2007;91:12 123504 .

7. Chen F. C., Chang S. C., He G., et al.Energy Transfer and Triplet Exciton Confinement in Polymeric Electrophosphorescent Devices. Journal of Polymer Science Part B; Polymer Physics. 2003;41:2126812690.

8. Ganzorig Chimed,FujihiraMasamichi.A Lithium Carboxylate Ultrathin Film on as Aluminium Cathode for Enhanced Electron Injection in Organic Electroluminescent Devices. Japanese Journal of Applied Physics.1999;38:111348-1350.

9. Samil Kho, SunyoungSohn, Donggeun Jung, et al.Characteristics of top-emission inverted organic light-emitting diodes with plasma-polymerized para-xylene cathode interfacial layers. Journal of Korean Physics Society.2005;46:51224-1227.

10. Michael M. Stephens, Edward D. Moorhead. An examination of the finite difference numerical approach the solution of electrochemically-induced diffusive transport at stationary solid cylinder electrodes. Journal ofEelctroanalytical Chemistry and Interfacial Electrochemistry.1984;164:117-26. 